Supporting information to

\title{
Perpendicular chain axis orientation in s-PS films: achievement by guest induced clathrate formation and maintenance after transitions toward helical and trans-planar polymorphic forms.
}

by Paola Rizzo*, Sofia Della Guardia, Gaetano Guerra

Dipartimento di Chimica, Università degli Studi di Salerno

Via S. Allende 84081 - Baronissi (Salerno) Italy

A better understanding of the orientation of the crystalline phases in s-PS films can be achieved by $\mathrm{X}$-ray patterns taken with incident beam perpendicular and parallel to the film plane, on a photographic cylindrical camera. ${ }^{22}$ The X-ray diffraction patterns collected with beam perpendicular to the film plane only show Debye Sherrer rings, indicating that there isn't any axial orientation in the film plane. For sake of simplicity, only X-ray diffraction patterns collected with incident beam parallel to the film plane, of a s-PS/TCE clathrate film (obtained by casting from TCE) and of $\gamma, \beta$ and $\alpha$ form films obtained by its annealing are reported in Figure A-D, respectively.

These X-ray diffraction patterns are similar to fiber patterns but they show the equatorial reflections located on the meridional line and the meridional reflections located on the equatorial line. This confirms, for all the considered guest induced clathrate phases, the presence of orientations with chain axes nearly perpendicular to the film plane, which are maintained after annealing procedures leading to $\gamma, \beta$ and $\alpha$ crystalline phases.

Figure . X-ray diffraction patterns collected with incident beam parallel to the film plane, by using a photographic cylindrical camera, recorded on a imaging plate and processed with a digital imaging reader, for: s-PS/TCE clathrate obtained by solution casting (A); $\gamma(\mathrm{B}) ; \beta(\mathrm{C})$ and $\alpha(\mathrm{D})$ form films obtained by annealing in different conditions a s-PS clathrate film. The corresponding X-ray diffraction patterns collected with an automatic powder diffractometer are reported in Figures 1B, 3C, 5C and 4C, respectively. 

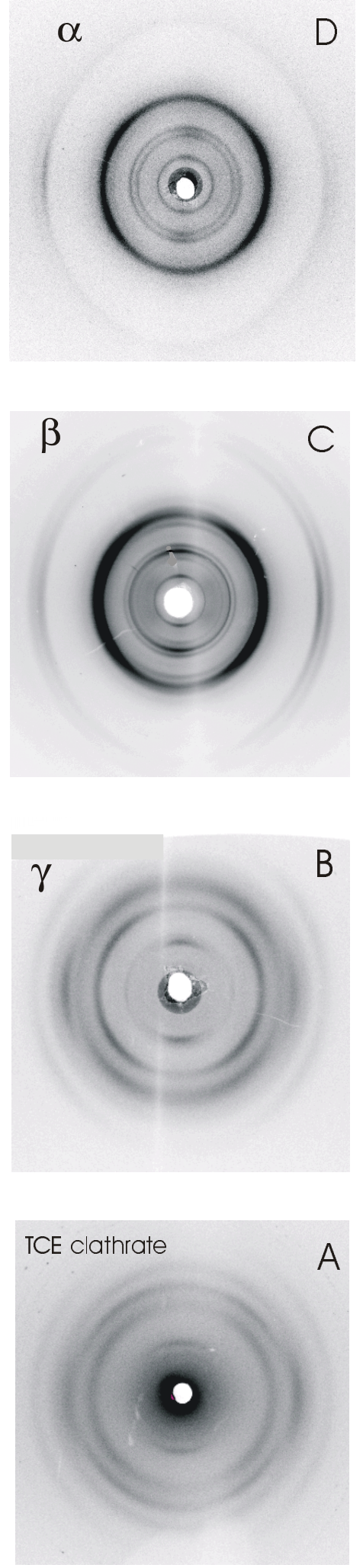

Figure 